
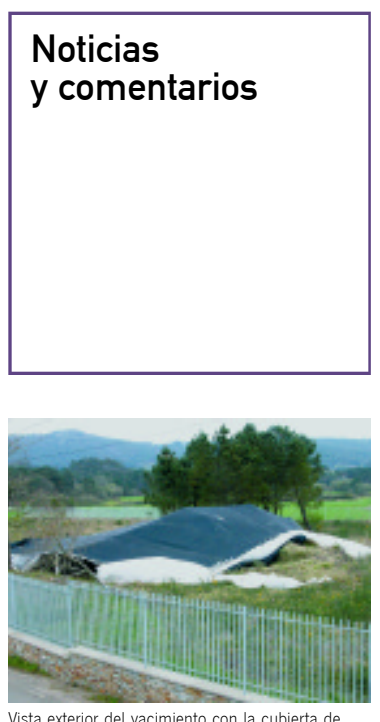

Vista exterior del yacimiento con la cubierta de plástico, solución utilizada en los años 80 y que continúa hoy en dia

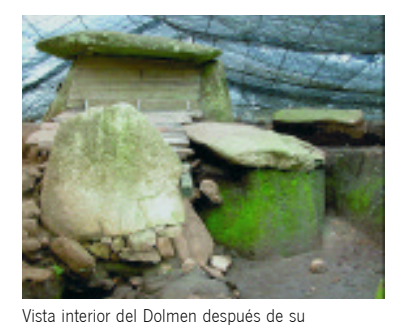
excavacior del Dolmen después de su excavación por M. Lestón

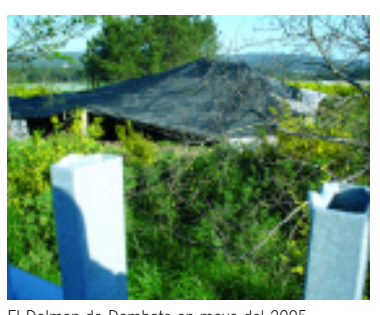

El Dolmen de Dombate en mayo del 2005

\title{
Se crea un observatorio para el seguimiento de la actividad arqueológica profesional
}

La línea de investigación que a continuación se propone se enmarca en un programa de investigación aplicada que tiene como objeto la introducción de la Arqueología, como tecnología, en las políticas de desarrollo sostenible. Partiendo de una perspectiva crítica de este último concepto y de la necesidad de una estrategia global para su implementación, consideramos que una visión integrada del medio natural y de los factores culturales, sociales y económicos que condicionan la actividad productiva en nuestra sociedad es una demanda prioritaria hoy en día.

En este sentido, el estudio se integra en el programa de Arqueología y Sociedad, que consiste en una línea de investigación colectiva en la que se busca la adaptación teórica, metodológica y técnica de la arqueología a la dinámica socioeconómica actual, en la que el Laboratorio de Arqueoloxía (IEGPS, CSIC-XuGa) trabaja desde 1992.

La iniciativa surge del contacto y compromisos del Laboratorio de Arqueoloxía con otras organizaciones europeas y del acuerdo ante la necesidad de poner en marcha iniciativas destinadas a coordinar la información referente a la arqueología aplicada en toda Europa, en cuanto práctica fuertemente atomizada por la dinamización del mercado y por las normativas reguladoras de cada país - región. Estas organizaciones son la EAA (Asociación Europea de Arqueólogos) y el EAC (Europae Archaelogicae Consilium).

\section{La Arqueología como práctica social}

El proyecto para la creación de un Observatorio de la Arqueología Profesional en España parte de las nuevas condiciones epistemológicas que surgen tras el declive del paradigma neopositivista. En este sentido, una parte de la investigación arqueológica debe reorientar sus esfuerzos hacia un nuevo objeto de estudio: la Arqueología en sí misma concebida como práctica social.

La adopción de un paradigma reflexivista, por tanto, implica que las preocupaciones epistemológicas tradicionales (objetividad, verdad), más vinculadas a un concepto básico de la arqueología como disciplina científica humanística, son relegadas a favor de una reflexión acerca de las condiciones sociales y características de la arqueologia, entendida como disciplina implicada en la producción y gestión de patrimonio arqueológico.

El objetivo general de este proyecto es intentar cubrir la creciente necesidad de un banco de datos tangible, que integre información variada sobre las actividades arqueológicas desarrolladas en el marco comercial, contractual y profesional de la Arqueología en España. Esta necesidad proviene del actual estado de desinformación y fragmentación de la Arqueología concebida como prestación de servicios (un ejemplo: no se sabe cuánto dinero mueve la Arqueología de servicio en España).

El Observatorio surge como un intento de centralizar la información y convertirla en conocimiento accesible y manipulable, tanto para arqueólogos profesionales como para investigadores, así como para gente interesada en la actividad arqueológica en España. Se trata de un proyecto que implica la adopción de una perspectiva sociológica, una sublínea de investigación orientada a la caracterización y evaluación de la actividad arqueológica profesional.

Se denomina "observatorio" porque se pretende realizar un seguimiento a largo plazo de las condiciones de trabajo de la Arqueología Profesional, lo que debería redundar, en definitiva, en:

$>$ Un mayor conocimiento de las condiciones reales del trabajo arqueológico en España.

> Una mejora colectiva en la calidad técnica de la disciplina, a través de la estandarización y normalización consensuada de criterios y procedimientos de actuación.

$>$ El incremento del flujo entre los distintos ámbitos de conocimiento que intervienen en este tipo de arqueología (histórico, técnico-instrumental, práctico), que debería favorecer una mayor integración de los datos generados.

> Una mayor capacidad de planificación y control por parte de la Administración de Patrimonio.

$>\mathrm{El}$ incremento del intercambio de información entre profesionales y, por consiguiente, su capacidad organizativa y de respuesta ante las dinámicas del mercado.

$>$ Una mayor accesibilidad a lo que la arqueología es y significa en el mundo actual, con vistas a una divulgación social real que, en definitiva, supondría un acortamiento de la gran distancia actualmente existente entre el trabajo de los expertos (en este caso, de los arqueólogos) y los agentes sociales implicados en los procesos de cambio socio-económico o modernización.

Teniendo en cuenta el contexto del estudio y sus objetivos generales, se consideran como objetivos especificos para una primera fase del trabajo las siguientes cuestiones:

> Concreción de la información considerada relevante: delimitación y censo de los sectores implicados, ya sean académicos (museos, universidades, grupos de investigación...), independientes (empresas, 
arqueólogos autónomos), o la Administración; caracterización del servicio; volumen de contratación; grado de difusión de resultados o flujos de información entre instancias.

> Diseño de una encuesta-tipo, en la que se recojan los campos de información relevante para ser realizada en las administraciones de Patrimonio de cada una de las Comunidades Autónomas españolas.

$>$ Realización de la encuesta y primera sistematización de la información.

\section{Plan de trabajo y resultados}

Para la consecución de estos objetivos se establece una metodología basada en distintos criterios. Mediante un primer momento de diseño, se pretende obtener una encuesta-tipo que recoja la información considerada relevante. Dicha encuesta, cuando esté dirigida a la administración, se orientará a la obtención de datos relativos al funcionamiento general de la actividad arqueológica de servicios en su ámbito correspondiente: volumen de trabajo global, parámetros de calidad, integración de resultados, etc. Dado que es la administración el ámbito prioritario de obtención de datos será aquí donde se concentren los mayores esfuerzos y donde la información será recabada de forma exhaustiva. Pero, como ya se especificó, cuando esté dirigida a profesionales independientes, la encuesta estará orientada a la obtención de los datos profesionales de los encuestados y a todas las circunstancias que pueden resultar de interés a la hora de formar parte del banco de datos. La metodología del muestreo deberá atender a la diversidad interna dentro de este sector: empresas, autónomos, trabajadores contratados.

Por último, cuando esté dirigida al sector académico, la encuesta deberá enfocarse, por un lado, a la obtención de datos referentes a la orientación de los planes de estudio hacia las salidas profesionales independientes. Por otro lado, se intentarían obtener datos relativos a la vinculación de instituciones como la Universidad, los museos y los centros de investigación respecto a la Arqueología Aplicada. En este sentido, habría dos ámbitos diferentes a los que atender: por una parte, el papel de la Academia en relación con la sistematización de resultados provenientes de la arqueología aplicada, es decir, si ésta participa de una actividad comercial de forma directa, a través de proyectos específicos, de forma indirecta, a través de la intervención en momentos pos- teriores (sistematización de datos, publicación de resultados, musealización de la cultura material...), o si no participa en modo alguno; en segundo lugar, el papel de la Academia respecto a la arqueología aplicada en tanto objeto de estudio en sí misma. Sobre todo en el primer ámbito, y al igual que en el caso anterior, habría que establecer una metodología de muestreo que posibilitase el abarcar la mayor diversidad posible en la muestra.

El momento de realización de la encuesta personalizada ocupará buena parte de esta primera fase de implementación del observatorio, ya que habrá que visitar las distintas administraciones de patrimonio en todo el Estado, además de entrar en contacto con una parte significativa de la arqueología profesional.

Posteriormente, se pasa a la sistematización de la información obtenida durante la encuesta, que cerrará esta primera fase del estudio. Las perspectivas para fases posteriores apuntan a una informatización de toda la información recabada y a la creación de una base de datos accesible en la web.

Entre los resultados previstos a largo plazo, se encuentra el desarrollo de un sistema de información conformado por datos relativos a la actividad arqueológica aplicada, formado tanto por cuestiones cuantitativas, tales como número de arqueólogos independientes, volumen de trabajo desarrollado, recursos generados..., como cuestiones cualitativas (resultados obtenidos, situación financiera de la Arqueología profesional, relaciones entre calidad del trabajo y situación real de los trabajadores).

De manera que este sistema tiene como función controlar un sector que se encuentra descontrolado, y ello aportaría datos para el análisis de la Arqueología Aplicada en tanto práctica social condicionada por la interacción entre lo político, lo cultural y lo económico, y sometida por tanto a tensiones entre intereses contrapuestos y juegos de poderconocimiento.

\section{María Cacheda Pérez}

Laboratorio de Arqueoloxia da Paisaxe

(Instituto de Estudios Galegos Padre

Sarmiento, CSIC-XuGa)

\section{Arte y naturaleza en ALBIAC}

Del 15 de julio al 15 de septiembre de 2006, Almería acoge la I Bienal Internacional de Arte Contemporáneo Cabo de Gata-Nijar (ALBIAC). El proyecto, que cuenta con la colaboración de las consejerías de Cultura y Medio Ambiente de la Junta de Andalucia, tendrá como marco de celebración un espacio protegido, el Parque Natural Cabo de Gata-Nijar.

Más información:

Web: www.XYZ.com 